\title{
ANEMIA E PERFIL DE LIPÍDEOS EM GESTANTES VIVENDO COM O VÍRUS DA IMUNODEFICIÊNCIA HUMANA
}

\section{Ana Teresa Mancini Pimenta}

Doutora pelo Departamento de Ginecologia e Obstetrícia da Faculdade de Medicina de Ribeirão Preto da Universidade de São Paulo(FMRP-USP), Ribeirão Preto(SP), Brasil.

E-mail: anateresa@usp.br

\section{Patricia Pereira dos Santos Melli}

Doutora; Médica assistente do Departamento de Ginecologia e Obstetrícia do Hospital das Clínicas da Faculdade de Medicina de Ribeirão Preto da Universidade de São Paulo(FMRP-USP), Ribeirão Preto(SP), Brasil.

\section{Conrado Milani Coutinho}

Doutor; Médico assistente do Departamento de Ginecologia e Obstetrícia do Hospital das Clínicas da Faculdade de Medicina de Ribeirão Preto da Universidade de São Paulo(FMRP-USP), Ribeirão Preto(SP), Brasil.

\section{Geraldo Duarte}

Doutor; Docente do Departamento de Ginecologia e Obstetrícia da Faculdade de Medicina de Ribeirão Preto da Universidade de São Paulo(FMRP-USP), Ribeirão Preto(SP), Brasil.

\section{Silvana Maria Quintana}

Doutora; Docente do Departamento de Ginecologia e Obstetrícia da Faculdade de Medicina de Ribeirão Preto da Universidade de São Paulo(FMRP-USP), Ribeirão Preto(SP), Brasil.
RESUMO: A infecção pelo HIV associa-se à anemia e às alterações no perfil de lipídeos de pessoas que vivem com o vírus. O objetivo deste estudo foi descrever a prevalência de anemia e as alterações nos níveis de lipídeos em gestantes que vivem com HIV/aids. O estudo foi do tipo coorte retrospectiva, realizado com 117 gestantes, em uso de terapia antirretroviral combinada, atendidas em ambulatório de prénatal no período de 2010 a 2012, utilizando-se de dados secundários. A prevalência de anemia foi de $45,6 \%$ e nenhuma gestante apresentou anemia severa. Observou-se aumento progressivo das dosagens de colesterol total $(p<0,001)$, HDL $(p=0,05)$, LDL $(p<0,001)$ e triglicérides $(p<0,001)$, com maior aumento dos níveis de triglicérides ao longo dos três trimestres gestacionais. O monitoramento da presença de anemia e dos níveis de lipídeos ao longo da gestação é essencial para a realização de intervenções precoces, quando necessário, evitando complicações gestacionais e desfechos indesejados.

PALAVRAS-CHAVE: Gestantes; HIV-1; Anemia; Lipídeos.

\section{ANEMIA AND LIPIDS PROFILE IN PREGNANT WOMEN LIVING WITH HIV}

ABSTRACT: HIV infection is associated with anemia and with changes in the profile of lipids in HIV-infected people. Current retrospective study described the prevalence of anemia and changes in lipid levels in pregnant women living with HIV/AIDS and was performed with 117 pregnant women who were using antiretroviral therapy in prenatal clinics, between 2010 and 2012, through secondary data. The prevalence of anemia reached $45.6 \%$, although no one had severe anemia. There was a progressive increase in total cholesterol $(p<0.001)$, HDL $(p=0.05)$, LDL $(p<0.001)$ and triglycerides $(p<0.001)$, with greater increase in triglycerides levels throughout pregnancy. Monitoring anemia and lipid levels during pregnancy is crucial for early interventions since undesirable complications and unwanted results are avoided.

KEY WORDS: Pregnant women; HIV-1; Anemia; Lipids.

\section{INTRODUÇÃO}

Dentre as modificações fisiológicas do organismo materno durante a gestação estão o aumento progressivo do volume plasmático e a elevação dos níveis de lipídeos. Tal hemodiluição fisiológica resulta na diminuição da concentração de hemoglobina, de hematócrito e da 
contagem de células vermelhas do sangue (SOMA-PILLAY et al., 2016), sendo denominada anemia fisiológica da gestação. A Organização Mundial de Saúde estabelece o limite de $11 \mathrm{~g} / \mathrm{dL}$ de hemoglobina, abaixo do qual se define anemia, independente da idade gestacional (WHO, 2008). Em populações com baixa ingestão de ferro e/ou má absorção do nutriente, o período gestacional, pela sua maior necessidade de ferro, oferece maior risco de desenvolvimento de anemia podendo associar-se a resultados obstétricos e neonatais adversos (WHO, 2014). Desse modo, o Ministério da Saúde recomenda para todas as gestantes, independente dos níveis de hemoglobina, a suplementação profilática de sulfato ferroso e ácido fólico a partir do início do prénatal até o terceiro mês pós-parto (BRASIL, 2013). Outro fator de risco associado à anemia é a infecção pelo HIV, sendo esta manifestação comumente observada em aproximadamente $69 \%$ das pessoas infectadas por este vírus (OMOREGIE et al., 2009).

As mudanças metabólicas na gestação culminam com o acúmulo materno de gordura, elevação de duas a três vezes das dosagens de triglicérides e aumentos menores no colesterol total, na lipoproteína de alta densidade (HDL) e na lipoproteína de baixa densidade (LDL), sobretudo durante o segundo e terceiro trimestre gestacionais (DIAREME et al., 2009; HERRERA; ORTEGASENOVILLA, 2010). Além disso, a infecção pelo HIV também influencia o metabolismo de lipídeos (diminuição da atividade da lípase de lipoproteínas, diminuição da produção de HDL e aumento do catabolismo de HDL) observando-se um perfil lipídico prejudicial ao indivíduo (CALZA et al., 2004; EL-SADR et al., 2005).

$\mathrm{O}$ uso da terapia antirretroviral combinada suprime a replicação viral reduzindo a carga viral para níveis indetectáveis, e permite a recuperação do sistema imunológico (FEENEY; MALLON, 2011) e sua utilização durante a gestação é uma das medidas mais eficazes na prevenção da transmissão vertical do HIV (TUOMALA et al., 2005; CARDOSO et al., 2010). No Brasil, desde 2006, o protocolo do Ministério da Saúde recomenda que todas as gestantes recebam terapia antirretoviral combinada (BRASIL, 2015), no entanto, toxicidades metabólicas são reconhecidas pelo seu uso, incluindo anemia e hiperlipidemia (CARR; COOPER, 2000; HERMAN; EASTERBROOK, 2001).
Com isso, o propósito deste estudo foi descrever a frequência de anemia e a variação dos níveis de colesterol, triglicérides, LDL e HDL nos três trimestres gestacionais em mulheres vivendo com HIV.

\section{METODOLOGIA}

Foi realizado um estudo do tipo coorte retrospectiva com gestantes que vivem com HIV, atendidas em serviço de pré-natal de hospital universitário no município de Ribeirão Preto, no interior do Estado de São Paulo, no período de 2010 a 2012. As gestantes incluídas assinaram o Termo de Consentimento Livre e Esclarecido (TCLE). Os dados clínico e laboratoriais foram coletados de prontuários médicos. No período de estudo, o esquema antirretroviral adotado era a associação de zidovudina e lamivudina juntamente com lopinavir e booster de ritonavir. Este trabalho é uma análise secundária de um estudo maior e foi aprovado pelo Comitê de Ética em Pesquisa do Hospital das Clínicas da Faculdade de Medicina de Ribeirão Preto da Universidade de São Paulo (processo $n^{0} 13411$ ).

\subsection{DEFINIÇÕES}

Valores normais de acordo com critérios laboratoriais:

Nível de hemoglobina $(\mathrm{Hb}) 11$ - 15,5 g/dL; glóbulos vermelhos 3,9 - 5,0x 10\% $\mu \mathrm{L}$; hematócrito (Ht) 35 - 45\%; volume corpuscular médio (VCM) 82 - 98FL; hemoglobina corpuscular média (HCM) 26 34PG; concentração de hemoglobina corpuscular média (CHCM) $31-36 \mathrm{~g} / \mathrm{dL}$; colesterol <200 mg/dL; HDL > 35 $\mathrm{mg} / \mathrm{dL}$; LDL $<130 \mathrm{mg} / \mathrm{dL}$ e triglicérides $<150 \mathrm{mg} / \mathrm{dL}$.

A anemia foi graduada em leve $(10,99-10,0 \mathrm{~g} /$ $\mathrm{dL})$, moderada $(9,9-7,0 \mathrm{~g} / \mathrm{dL})$ e severa $(<7,0 \mathrm{~g} / \mathrm{dL})$.

\subsection{Análise estatística}

Foram realizados modelos de regressão linear de efeitos mistos. Foi checada a distribuição dos resíduos por meio de gráficos de normalidade e de dispersão entre o resíduo versus o predito. Foram considerados significativos valores de $\mathrm{p}$ inferiores a 0,05 . Foi utilizado o programa SAS, versão 9.3 e a análise foi implementada no Proc Mixed. 


\section{RESULTADOS}

O estudo incluiu 117 gestantes que vivem com HIV, com idade mediana de 29 anos (Tabela 1).

Tabela 1. Características das gestantes infectadas pelo HIV $(\mathrm{n}=117)$. HCFMRP-USP, 2010-2012

\begin{tabular}{l|c}
\hline Características & \\
\hline Idade, anos* & $29(15-43)$ \\
Tempo de diagnóstico da infecção HIV, anos* & $2(0-17)$ \\
Uso de antirretrovirais, n (\%) & $52(44,4)$ \\
Nä̈ve & $65(55,6)$ \\
Uso prévio de ARV & \\
Início do pré-natal - trimestre gestacional, n & \\
(\%) & $34(29,1)$ \\
$1^{\circ}$ & $50(42,7)$ \\
$2^{\circ}$ & $33(28,2)$ \\
$3^{\text {o }}$ & $5853(<50-$ \\
Carga viral no início do pré-natal, cópias/ mL* & $323.319)$ \\
& $414,5(8-1.189)$ \\
CD4+ no início do pré-natal, células/mm3* & $806(240-2.626)$ \\
\hline CD8+ no início do pré-natal, células/mm3* & \\
\hline * Valores representados em mediana; (amplitude) &
\end{tabular}

* Valores representados em mediana; (amplitude)
Destas gestantes, $52(44,4 \%)$ nunca utilizaram antirretrovirais e 65 (55,6\%) já fizeram uso de antirretrovirais previamente. Destas, 36 utilizaram antirretrovirais de maneira profilática e 29 utilizavam antirretrovirais para seu tratamento quando engravidaram. As gestantes que não estavam em uso de antirretrovirais iniciaram o uso a partir da $14^{\mathrm{a}}$ semana de gestação. Quanto à classificação da infecção pelo HIV, de acordo com o Center for Disease Control and Prevention (CDC, 1993), 67,5\% estavam classificadas como assintomáticas.

No primeiro trimestre gestacional, 22,2\% das gestantes apresentaram anemia leve, normocítica e normocrômica. No segundo trimestre, $29,6 \%$ das gestantes apresentaram anemia leve e $8,5 \%$ anemia moderada (total de 38\% apresentaram anemia). Das mulheres com anemia, 59,3\% eram normocíticas e 29,6\% macrocíticas. No terceiro trimestre, $25,8 \%$ apresentaram anemia leve e $12,9 \%$ anemia moderada (total de 38,7\% apresentaram anemia). Destas, $40 \%$ eram macrocíticas e $50 \%$ normocíticas. No total, $45,6 \%$ das gestantes apresentaram anemia em pelo menos um trimestre gestacional. Na Tabela 2 estão as médias dos resultados dos eritrogramas nos três trimestres gestacionais.

Tabela 2. Distribuição das gestantes infectadas pelo HIV de acordo com resultados de eritrograma. HCFMRP-USP, $2010-2012$

\begin{tabular}{|c|c|c|c|c|c|c|}
\hline \multirow[t]{2}{*}{ Exames } & \multicolumn{2}{|c|}{$1^{\circ}$ trimestre } & \multicolumn{2}{|c|}{$2^{\circ}$ trimestre } & \multicolumn{2}{|r|}{$3^{\circ}$ trimestre } \\
\hline & $\mathbf{n}$ & média $\pm d p$ & $\mathbf{n}$ & média $\pm d p$ & $\mathbf{n}$ & média $\pm d p$ \\
\hline $\mathrm{Hb}(\mathrm{g} / \mathrm{dL})$ & 27 & $11,89 \pm 1,02$ & 73 & $11,12 \pm 1,76$ & 93 & $11,33 \pm 1,57$ \\
\hline Glóbulos vermelhos $(106 / \mu \mathrm{L})$ & 27 & $3,74 \pm 0,44$ & 73 & $3,64 \pm 0,57$ & 93 & $3,41 \pm 0,51$ \\
\hline Ht (\%) & 27 & $35,74 \pm 2,93$ & 73 & $34,52 \pm 4,03$ & 93 & $34,11 \pm 3,64$ \\
\hline VCM (FL) & 25 & $98,53 \pm 11,16$ & 70 & $96,12 \pm 11,68$ & 80 & $100,79 \pm 13,14$ \\
\hline $\mathrm{HCM}(\mathrm{PG})$ & 25 & $32,70 \pm 4,24$ & 70 & $31,36 \pm 4,42$ & 80 & $33,14 \pm 4,51$ \\
\hline CHCM (g/dL) & 25 & $33,12 \pm 1,08$ & 70 & $32,56 \pm 1,19$ & 80 & $32,89 \pm 1,18$ \\
\hline
\end{tabular}

O lipidograma de todas as gestantes no primeiro trimestre gestacional estava dentro dos valores considerados normais. No segundo trimestre, 35,5\% das gestantes apresentaram dosagem de colesterol acima do esperado $(6,5 \% \geq 240)$ e $47,6 \%$ apresentaram triglicérides acima dos valores esperados $(21,3 \%$ entre 200 e 499), sendo que $66,7 \%$ daquelas com colesterol aumentado também apresentaram valores de triglicérides aumentados.Dois valores alterados no lipidograma foram observados em 35,5\% das mulheres.
No terceiro trimestre gestacional, $75,4 \%$ apresentaram dosagem de colesterol acima do esperado $(32,3 \% \geq 240)$ e $87,5 \%$ apresentaram triglicérides acima dos valores esperados (52,3\% entre 200 e 499). Daquelas com valores elevados de colesterol, 89,8\% também apresentaram valores de triglicérides aumentados. $\mathrm{Na}$ Tabela 3 estão as médias dos resultados do lipidograma nos três trimestres gestacionais De todas as gestantes que realizaram o exame de lipidograma, $70,8 \%$ apresentaram pelo menos dois valores alterados. Em 13\% das gestantes 
Tabela 3. Distribuição das gestantes infectadas pelo HIV de acordo com resultados do lipidograma. HCFMRP-USP, 2010-2012

\begin{tabular}{lcccccc}
\hline Exames & \multicolumn{2}{c}{$\mathbf{1}^{\mathbf{0}}$ trimestre } & \multicolumn{2}{c}{$\mathbf{2}^{\mathbf{0}}$ trimestre } & \multicolumn{2}{c}{$\mathbf{3}^{\mathbf{0}}$ trimestre } \\
\hline & $\mathbf{n}$ & $\mathbf{m e ́ d i a} \pm \mathbf{d p}$ & $\mathbf{n}$ & média $\pm \mathbf{d p}$ & $\mathbf{n}$ & média $\pm \mathbf{d p}$ \\
\cline { 2 - 7 } Colesterol (mg/dL) & 20 & $151,20 \pm 26,57$ & 62 & $189,32 \pm 33,32$ & 65 & $232,91 \pm 58,41$ \\
HDL (mg/dL) & 20 & $44,35 \pm 10,95$ & 55 & $49,38 \pm 13,26$ & 60 & $52,43 \pm 12,09$ \\
LDL (mg/dL) & 20 & $86,60 \pm 17,79$ & 55 & $106,05 \pm 24,51$ & 54 & $133,13 \pm 49,20$ \\
Triglicérides (mg/dL) & 20 & $101,05 \pm 47,05$ & 61 & $155,70 \pm 66,84$ & 64 & $235,70 \pm 92,1$ \\
\hline
\end{tabular}

os valores de LDL apresentaram-se altos (160-189) e em $5,6 \%$ delas, muito alto $(\geq 190)$.

Do primeiro para o segundo trimestre de gestação houve aumento de $25,2 \%$ na média dos níveis de colesterol; $11,3 \%$ do HDL; $22,5 \%$ do LDL e $54,1 \%$ dos triglicérides. Do segundo para o terceiro trimestre houve aumento de $23 \%$ na média dos níveis de colesterol; 6,2\% do HDL; $25,5 \%$ do LDL e $51,4 \%$ dos triglicérides. Do primeiro para o terceiro trimestre houve aumento de $54 \%$ na média dos níveis de colesterol; $18,2 \%$ do HDL; $53,7 \%$ do LDL e $133,3 \%$ dos triglicérides. Figura 1.
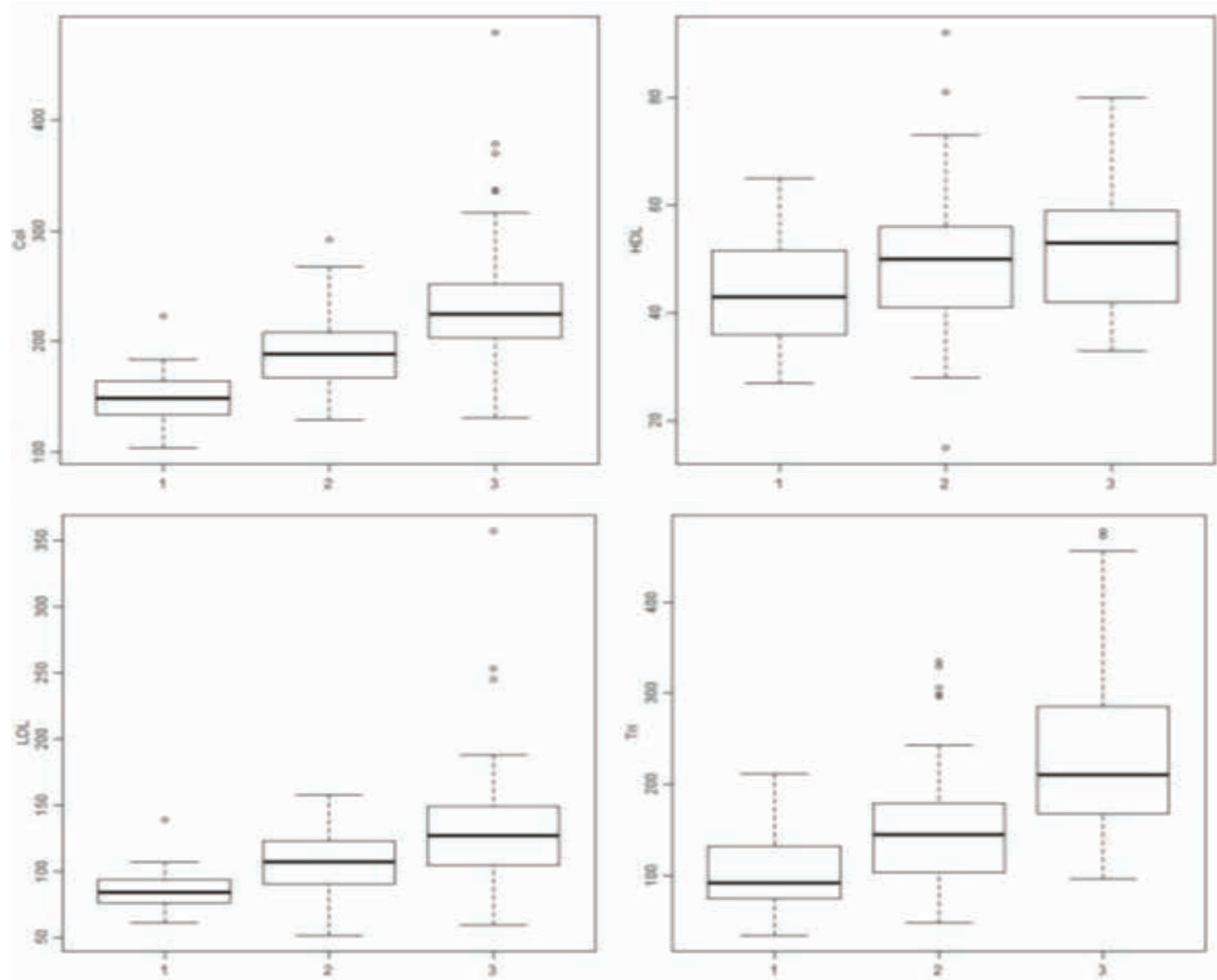

Figura 1. Boxplot das dosagens de colesterol (Col), HDL, LDL e triglicérides (Tri) nos três trimestres gestacionais, respectivamente 1, 2 e 3. 


\section{DISCUSSÃO}

Neste estudo, 45,6\% das gestantes que vivem com HIV apresentaram anemia $(\mathrm{Hb}<11 \mathrm{~g} / \mathrm{dL})$ em pelo menos um trimestre gestacional. A prevalência variou de $22,2 \%$, no primeiro trimestre a $38,7 \%$ no terceiro trimestre, com menor média de dosagens de hemoglobina no segundo trimestre. Outros estudos relataram a prevalência de anemia entre gestantes que vivem com HIV de 5,3\% ( $\mathrm{Hb} \leq 9,4 \mathrm{~g} / \mathrm{dL}$ ) na Tailândia (AREECHOKCHAI et al., 2009), 19,1\% no Brasil (KONOPKA et al., 2010), 23,8\%(Hb <10 g/dL) em países da América Latina e Caribe (CERIOTTO et al., 2008), 38,7\%(Hb<10 g/dL) na Índia (SINHA et al., 2007), 57,3\% na Nigéria (ADESINA et al., 2011) e 61,6\% $(\mathrm{Hb}<11 \mathrm{~g} / \mathrm{dL})$ no Brasil (BRANDÃO et al., 2011).

Um estudo de revisão sistemática sobre o uso de terapia antirretroviral combinada observou que seu uso produz aumento da concentração de hemoglobina e diminuição da prevalência de anemia (BELPERIO; RHEW, 2004). Em outro estudo, o uso de zidovudina não foi considerado um fator agravante da anemia nas grávidas em uso deste antirretroviral, no entanto a prevalência de anemia foi maior entre as gestantes que vivem com HIV que naquelas gestantes não infectadas (SINHA et al., 2007). A zidovudina é considerada associada à toxicidade hematológica, principalmente a mielossupressão (VOLBERDING et al., 2004). Desse modo, há estudos que apontam maior propensão de gestantes em uso de antirretrovirais apresentarem anemia (HERMAN; EASTERBROOK, 2001; TUOMALA et al., 2005).

O porcentual de gestantes deste estudo com baixa dosagem de hemoglobina foi crescente no decorrer dos três trimestres gestacionais, contudo nenhuma apresentou anemia severa. Porém, visto que a hemodiluição é mais importante até a $18^{\mathrm{a}}-20^{\mathrm{a}}$ semana gestacional (SOMA-PILLAY et al., 2016) a fisiopatologia da infecção pelo HIV deve ser levada em consideração (VOLBERDING et al., 2004).

A prevalência de alteração na dosagem de lipídeos foi maior no terceiro trimestre gestacional, observandose aumento da porcentagem de gestantes com alterações ao longo da gestação. Valores no lipidograma estavam alterados em $20 \%$ das gestantes no primeiro trimestre gestacional, em 59,7\% no segundo trimestre e em $95,4 \%$ no terceiro trimestre. Verificou-se maior aumento de triglicérides ao longo da gestação e menor aumento de HDL.

A gestação é uma causa secundária para o aumento de triglicérides e LDL, porém, a utilização de terapias antirretrovirais, contendo inibidores da protease, é também considerada causa secundária para o aumento de colesterol total, LDL e triglicérides (JELLINGER et al., 2012). Vários estudos já demonstraram que gestantes em uso de terapia antirretroviral, contendo inibidores da protease, apresentam aumento progressivo de lipídeos ao longo dos três trimestres de gestação, com aumento significativo na média de colesterol total, triglicérides e LDL (GUTIÉRRREZ et al., 2003, FLORIDIA et al., 2006). Em estudo prospectivo, El Beitune e colaboradores (2003) acompanharam três grupos de gestantes: 1) infectadas pelo HIV em uso de zidovudina, 2) infectadas pelo HIV em uso de zidovudina + lamivudina + nelfinavir e 3) controle. Foi observado elevação dos níveis de colesterol nos três grupos, porém elevação de LDL foi constatada apenas nas gestantes infectadas pelo vírus.

$\mathrm{O}$ aumento progressivo dos níveis de colesterol e triglicérides ao longo da gestação é fisiologicamente normal. Estudo realizado por Monafa e colaboradores (2012) observou que alterações no perfil lipídico de gestantes que vivem com HIV e em uso de antirretrovirais era significativamente menor que em gestantes não infectadas pelo vírus. O presente estudo mostrou maior variação na alteração dos níveis lipídicos, com exceção do HDL, em comparação com aquele estudo. O trabalho de Diareme e colaboradores (2009), realizado com gestantes saudáveis, mostrou níveis de colesterol e de LDL mais elevados nos três trimestres, níveis de HDL mais elevados nos dois últimos trimestres e triglicérides mais baixos durante a gravidez que em gestantes que vivem com HIV do presente estudo e daquele realizado por Monafa et al. (2012). Floridia e colaboradores (2006) relataram níveis significativamente mais elevados de triglicérides em todos os trimestres gestacionais em grávidas utilizando terapias antirretrovirais contendo inibidores da protease que em grávidas, utilizando terapias sem conter os inibidores de protease.

Terapias antirretrovirais de alta potência (HAART) podem causar complicações metabólicas como 
aumento de triglicérides e colesterol e também anemia (HERMAN; EASTERBROOK, 2001). No entanto, o início precoce dos antirretrovirais é crucial para reduzir a carga viral para níveis indetectáveis no parto, o que representa um dos pilares da prevenção da transmissão vertical (ALCÂNTARA et al., 2012). A recomendação é que todas as gestantes infectadas pelo HIV façam uso de terapia antirretroviral, independente do estado clínico, virológico ou imunológico da mulher. Porém, infecção por HIV, gestação e uso da combinação de antirretrovirais são fatores independentes associados a mudanças nos lipídeos plasmáticos (FLORIDIA et al., 2006).

A gestação é um evento que por si só provoca alterações fisiológicas na mulher favorecendo a diminuição das dosagens de hemoglobina e aumento dos valores de lipídeos. A infecção pelo HIV parece acentuar tais eventos adversos. Mas, é preciso destacar que a utilização de antirretrovirais para a prevenção da transmissão vertical do vírus é bastante efetiva. Assim, o início precoce do pré-natal para realização de exames, orientações e tratamentos de infecções juntamente com o manejo desses eventos adversos durante a gestação é crucial para a manutenção da saúde da mulher e da saúde de seu filho.

\section{CONCLUSÃO}

Embora a infecção pelo HIV e o uso de terapia antirretroviral combinada, nenhuma gestante apresentou anemia severa e no perfil de lipídeos séricos os aumentos podem ser considerados dentro do esperado. Conclui-se que o monitoramento dos níveis de hemoglobina e de lipídeos ao longo da gestação em mulheres que vivem com HIV é essencial para a realização de intervenções precoces, quando necessário, evitando-se complicações gestacionais e desfechos indesejados.

\section{REFERÊNCIAS}

ADESINA, O. et al. Risk of anaemia in HIV positive pregnant women in Ibadan, south west Nigeria. Afr $\mathbf{J}$ Med Sci, v. 40, n.1, p. 67-73, 2011.
ALCÂNTARA, K. C. etal. HIV-1 mother-to-child transmission and drug resistance among Brazilian pregnant women with high access to diagnosis and prophylactic measures $\mathbf{J}$ Clin Virol, n. 54, n.1, p.15-20, 2012.

AREECHOKCHAI, D. et al. Pregnancy outcomes among HIV-infected women undergoing antiretroviral therapy. Open AIDS J., v. 3, p. 8-13, 2009.

BELPERIO, P. S.; RHEW, D. C. Prevalence and outcomes of anemia in individuals with human immunodeficiency virus: a systematic review of the literature. Am J Med, v. 116, n. Suppl 7A, p. 27S-43S, 2004.

BRANDÃO, T. et al. Características epidemiológicas e nutricionais de gestantes vivendo com HIV. Rev Bras Ginecol Obstet, v. 33, n. 8, p. 188-195, 2011.

Brasil. Ministério da Saúde. Programa Nacional de suplementação de ferro: manual de condutas gerais. Brasília, 2013.

BRASIL. Ministério da Saúde. Protocolo clínico e diretrizes terapêuticas para prevenção da transmissão vertical de HIV, sífilis e hepatites virais. Brasília: inistério da Saúde, 2015.

CALZA, L.; MANFREDI, R.; CHIODO, F. Dyslipidaemia associated with antiretroviral therapy in HIV-infected patients. J Antimicrob Chemother, v. 53, p.10-14, 2004.

CARDOSO, L. P. et al. HIV-1 primary and secondary antiretroviral drug resistance and genetic diversity among pregnant women from central Brazil. J Med Virol, v. 82, n. 3, p. 351-317, 2010.

CARR, A.; COOPER, D. A. Adverse effects of antiretroviral therapy. Lancet, v. 356, p. 1423-1430, 2000.

CERIOTTO, M. et al. Laboratory abnormalities among HIV-1-infected pregnant women receiving antiretrovirals in Latin America and the Caribbean. AIDS Patient Care STD, v. 22, n. 3, p. 167-171, 2008.

DIAREME, M. et al. Lipid profile of healthy women during normal pregnancy. J M B, v. 28, p. 152-160, 2009.

EL BEITUNE, P. et al. O uso de anti-retrovirais em gestantes modifica o perfil lipídico? Rev Bras Ginecol Obstet, v. 25, n.8, p.593-598, 2003.

EL-SADR, W. M. et al. Effects of HIV disease on lipid, glucose and insulin levels: results from a large antiretroviral-naïve 
cohort. HIV Med, v. 6, n.2, p. 114-121, 2005.

FEENEY, E. R.; MALLON, P. W. G. HIV and HAARTassociated dyslipidemia. Open Cardiovasc Med, v. 5, p. 49-63, 2011.

FLORIDIA, M. et al. Lipid profile during pregnancy in HIV-infected women. HIV Clin Trials, v. 7, n. 4, p. 184193, 2006.

GUTIÉRREZ, F. et al. Lopinavir plasma concentration and changes in lipid levels during salvage therapy with lopinavir/ ritonavir-containing regimens. J Acquir Immune Defic Syndr, v. 33, n.5, p. 594-600, 2003.

HERMAN, J. S.; EASTERBROOK, P. J. The metabolic toxicities of antiretroviral therapy. Int J STD AIDS, v. 12, p.555-564, 2001.

HERRERA, E.; ORTEGA-SENOVILLA, H. Maternal lipid metabolism during normal pregnancy and its implication to fetal development. Clin Lipidol, v. 5, n. 6, p. 899-911, 2010 .

JELLINGER, P. S. et al. American Association of Clinical Endocrinologists' Guidelines for Management of Dyslipidemia and Prevention of Atherosclerosis. Endocr Pract, v. 18, n. Suppl 1, p.1-78, 2012.

KONOPKA, C. K. et al. Perfil clinico e epidemiológico de gestantes infectadas pelo HIV em um serviço do sul do Brasil. Rev Bras Ginecol Obstet, v. 32, n.4, p. 184-190, 2010.

MONAFA, P. O. et al. Assessment of Lipid Profile in HIV Seropositive Pregnant Women attending Ante-Natal Clinic in Nnamdi Azikiwe University Teaching Hospital, Nnewi, Anambra State, Nigeria. Orient Journal of Medicine, $v$. 24, n. 3-4, p. 40-44, 2012.

OMOREGIE, R. et al. Prevalence of anaemia among HIVinfected patients in Benin city, Nigeria. Tanzan J Health Res, v. 11, n.1, p. 1-4, 2009;

SINHA, G. et al. Clinically significant anemia in HIVinfected pregnant women in India is not a major barrier to zidovudine use for prevention of maternal-to-childtransmission. J Acquir Immune Defic Syndr, v. 45, n.2, p. 210-217, 2007.
SOMA-PILLAY, P. et al. Physiological changes in pregnancy. Cardiovasc J Afr, v. 27, n.2, p. 89-94, 2016.

TUOMALA, R. E. et al. Improved obstetric outcomes and few maternal toxicities are associated with antiretroviral therapy, including highly active antiretroviral therapy during pregnancy. J Acquir Immune Defic Syndr, v. 38, n.4, p.449-473, 2005.

VOLBERDING, P. A. et al. Anemia in HIV infection: clinical impact and evidence-based management strategies. Clin Infect Dis, v. 38, n.10, p. 1454-1363, 2004.

WHO. World Health Organization. WHA global nutrition targets 2025: anaemia policy brief. Disponível em: $<$ http://www.who.int/nutrition/topics/globaltargets anaemia_policybrief.pdf?ua $=1 \& u a=1>$. Acesso em: 04 jul. 2016.

WHO. World Health Organization. Worldwide prevalence of anaemia 1993 - 2005. Disponível em: < http:// whqlibdoc.who.int/publications/2008/9789241596657 eng.pdf > . Acesso em: 18 jul. 2013.

Recebido em: 22 de novembro de 2016 Aceito em: 31 de março de 2017 